\title{
Comment attirer le gibier? Pratique du jeûne et récitation de chants magiques dans les activités cynégétiques wampis (jivaro)
}

How to attract game? Fasting and performing magical songs in Wampis hunting activities (Jivaro)

¿Cómo atraer la presa? Práctica del ayuno y enunciación de cantos mágicos en las actividades cinegéticas wampis (jivaro)

\section{Paul Codjia}

\section{OpenEdition \\ Journals}

Édition électronique

URL : https://journals.openedition.org/jsa/15172

DOI : 10.4000/jsa. 15172

ISSN : $1957-7842$

\section{Éditeur}

Société des américanistes

\section{Édition imprimée}

Date de publication : 15 décembre 2017

Pagination : 81-109

ISSN : 0037-9174

Référence électronique

Paul Codjia, "Comment attirer le gibier? Pratique du jeûne et récitation de chants magiques dans les activités cynégétiques wampis (jivaro)», Journal de la Société des américanistes [En ligne], 103-2 | 2017 mis en ligne le 15 décembre 2017, consulté le 26 septembre 2022. URL : http:// journals.openedition.org/jsa/15172 ; DOI : https://doi.org/10.4000/jsa.15172 


\title{
Comment attirer le gibier? Pratique du jeûne et récitation de chants magiques dans les activités cynégétiques wampis (jivaro)
}

\author{
Paul CoDJIA *
}

\begin{abstract}
Pour favoriser les rencontres avec le gibier, les chasseurs wampis jeûnent et énoncent des chants magiques anen. La performativité de ces chants repose sur la capacité du chasseur à réaliser un travail affectif sur lui-même: selon les Wampis, si le chanteur fait l'expérience sensible de la relation avec l'allocutaire décrite dans l'incantation, alors ce dernier l'éprouvera également. Le chasseur s'applique ainsi à « penser bien », shir anentaimat, la scène décrite dans le texte des anen. L'objectif de ces deux pratiques est de parvenir à manipuler les pensées et les émotions de l'animal de telle sorte qu'il agisse en faveur de la réussite cynégétique. Pour être efficace, le chant est subvocalisé car l'intention manipulatrice doit rester cachée. À travers une analyse pragmatique et sémantique de ces chants, cet article met en lumière les dispositifs rituels et linguistiques qui aident le chasseur à établir la relation désirée avec la proie. [Mots-clés: Wampis, chant magique, jeûne, chasse, pragmatique, affect.]
\end{abstract}

How to attract game? Fasting and performing magical songs in Wampis hunting activities (Jivaro). Wampis hunters fast and perform anen magical songs to attract game. The performativity of these songs rests upon the hunter's ability to realize an affective work on himself: from the Wampis' point of view, if the singer gets a bodily experience of the relationship with the addressee described by the spell, so the latter will also feel it. The hunter has to "think intensely", shir anentaimat, the scene depicted by the text of the anen. The purpose is to manipulate the animal's thoughts and emotions so that it may act in favour of a propitious hunting outcome. To be effective, the manipulative intent must remain hidden from the game, which is why anen are subvocalized. By a pragmatic and semantic analysis of these magical songs, this article aims to reveal the ritual and linguistic devices which allow the hunter to establish the desired relationship with the prey. [Key words: Wampis, magical song, fasting, hunting, pragmatic, affect.]

¿Cómo atraer la presa? Práctica del ayuno y enunciación de cantos mágicos en las actividades cinegéticas wampis (jivaro). Los cazadores Wampis ayunan y

*LAS, EHESS, Paris [paul.codjia@hotmail.fr]. 
enuncian cantos mágicos anen para atraer las presas. La performatividad de estos cantos depende de la capacidad del cazador de efectuar un trabajo afectivo sobre sí mismo: para los Wampis, si el cantante experimenta la relación con el destinatario descrita en la canción, este último también la sentirá. El cazador se esfuerza en "pensar bien", shir anentaimat, la escena descrita en los anen. El objetivo es el de manipular los pensamientos y emociones del animal para que éste se aproxime y la caza sea un éxito. El canto es entonces subvocalizado para evitar que el animal perciba la trampa. El análisis de la pragmática y semántica de estos cantos mágicos permitirá revelar los dispositivos rituales y lingüísticos que ayudan al cazador a establecer la relación deseada con la presa. [Palabras claves: Wampis, cantos mágicos, ayuno, caza, pragmática, afecto.]

Un matin de janvier 2014, alors que le soleil n'est pas encore apparu au-dessus de l'épaisse canopée amazonienne, nous partons en petit groupe d'une dizaine de personnes, hommes et femmes, à jeun, en direction d'un camp installé par une petite entreprise d'entretien de l'oléoduc nord-péruvien. Sans prévenir les habitants, l'entreprise a commencé des travaux sur la portion du pipe-line qui traverse le territoire titularisé de la communauté wampis ${ }^{1}$ de Mayuriaga, située sur les rives d'un petit affluent éponyme de la rivière Morona. Ceux-là y voient une violation de leur espace et réclament en conséquence que l'entreprise emploie une main-d'œuvre locale. Le camp se trouve à environ dix kilomètres du village. Outre l'espoir de s'assurer une source de revenus à l'issue de l'expédition, mes compagnons ont aussi celui de rencontrer du gros gibier qui s'éloigne de plus en plus des centres peuplés. Les hommes s'arment de leur fusil en vue d'une éventuelle partie de chasse. Le meilleur moyen de nous rendre au camp est de

1. Les Wampis, ou Huambisa en espagnol, appartiennent au groupe linguistique jivaro regroupant les Achuar, les Shuar, les Shiwiar et les Awajún dont les langues sont mutuellement intelligibles. Ces cinq populations forment un sous-groupe d'un ensemble plus large incluant les voisins orientaux des Wampis, les Shapra et les Candoshi, avec lesquels ils partagent de nombreux traits culturels. Leurs langues sont cependant très différentes. Le territoire wampis est situé au nord de l'Amazonie péruvienne à cheval sur les régions Amazonas à l'Ouest et Loreto à l'Est, et s'arrête à la frontière équatorienne. Ils habitent dans des communautés légalisées sous le régime de titres de propriété communs par la loi de Comunidades Nativas de 1974. Une publication du ministère de la Culture péruvien de 2015 estime, à partir des données du dernier recensement national de 2007 (Instituto Nacional de Estadística e Informática, INEI), à environ 10000 le nombre d'habitants des communautés wampis. Un chiffre qu'il faut revoir désormais à la hausse. Le nom Wampis provient très probablement du mot wampi, le poisson Salminus iquitensis qui a la réputation d'être rapide et difficile à pêcher. Les Wampis m'ont rapporté que ce nom leur avait été donné par les Awajún avec lesquels ils entretenaient des conflits guerriers de chasse aux têtes. Les Wampis s'auto-dénommaient shuar (personne), mais le nom Wampis s'est stabilisé et est devenu aujourd'hui l'ethnonyme adopté par l'administration péruvienne. La langue wampis possède un alphabet officiel depuis 2013, que j'utilise conjointement avec les traducteurs pour écrire les chants anen. Les Wampis sont traditionnellement des chasseurs, des pêcheurs et des horticulteurs. 
marcher sur l'oléoduc d'un diamètre de quarante centimètres afin d'éviter de mettre les pieds dans les zones marécageuses. Sur le chemin du retour, nous sommes exténués par la marche en équilibre sous le soleil brûlant qui chauffe le métal du tube. Nous n'avons pas déjeuné avant de partir et, pour essayer de calmer la soif et la faim, buvons à intervalles réguliers quelques gorgées de nijamanch, la boisson de manioc fermentée. Je marche devant mes amis Elias et Felipe qui, depuis quelque temps, ont cessé de parler pour se concentrer sur les bruits provenant de la forêt. Ils me disent que c'est le moment idéal pour trouver du gibier. Le matin même, je m'étais éveillé en même temps que Felipe et il m'avait raconté son rêve de chasse de bon augure. Il avait rêvé qu'il se faisait menacer par un homme avec une machette, signe qu'il rencontrerait dans la journée un pécari, paki en wampis. Il en était persuadé. Occupé à me couvrir la tête avec un t-shirt pour éviter une insolation, j'ai l'impression d'être le seul à me sentir au bord du malaise. Je serai détrompé plus tard par un de nos compagnons subitement pris de vomissements. Soudain, un jeune qui avait pris de l'avance revient vers nous en courant et nous dit de ne plus faire de bruit, qu'à une trentaine de mètres sur notre droite, il a entendu des paki. Le regard de mes compagnons change, surtout celui de Felipe. Ragaillardis par la nouvelle, mes amis sautent à terre, se dispersent et pénètrent dans la forêt. Je décide de suivre Elias tant bien que mal. Il n'a pas pris son fusil mais souhaite tout de même participer à l'assaut. Les pieds pris dans la végétation, j'entends des coups de feu. Nous nous enfonçons un peu plus dans la forêt puis, perdus, nous décidons de retourner vers l'oléoduc. C'est alors qu'apparaît Felipe, fier et souriant, les épaules chargées par le corps inerte d'un gros pécari. Il finira le trajet en portant la bête jusqu'à la communauté. Arrivé chez lui, Felipe entreprend de vider, de dépecer et de découper l'animal à la machette. Il me fait remarquer que son rêve s'est réalisé. Que grâce à son rêve, il a pensé toute la journée au pécari qu'il allait rencontrer et que de tanto sufrimiento, de « tant souffrir », le rêve s'est réalisé. Cette anecdote illustre de manière exemplaire le processus complexe de travail affectif que les Wampis effectuent sur eux-mêmes en vue de la réussite cynégétique. Deux éléments sont importants: souffrir, notamment par le jeûne, et se concentrer, penser intensément à l'animal. Ce deuxième élément revêt un sens tout particulier lorsqu'on sait que le chasseur peut réciter mentalement une incantation $a$ anen $^{2}$ afin d'attirer sa proie. Selon les Wampis, en énonçant sotto voce ce chant magique, le chanteur parvient à penser et ressentir la relation désirée avec l'animal en vue de manipuler les pensées,

2. Les Wampis prononcent «anen » mais la forme du mot avant élision, telle qu'elle est révélée par les analyses morphosyntaxiques, est anenta. Le mot anenta est à la fois le substantif signifiant « chant magique » et le verbe « chanter un chant magique ». Le verbe anenta- apparaît cependant toujours avec l'applicatif -ru, anentru-, qui donne le sens de « chanter un anen à quelqu'un ». 
les émotions et le comportement de ce dernier. Pour que l'anen soit efficace, sa récitation doit se faire de préférence le ventre vide et être subvocalisée afin que le destinataire ignore qu'un charme lui est adressé.

On trouve le même type de chants chez les Shuar (Harner 1972 ; Napolitano 1988; Amadio 1988), les Awajún (Brown 1984, 1985), les Achuar (Taylor et Chau 1983; Descola 1986, 1993a; Taylor 2000) et les Candoshi (Surrallés 2003). Ce sont des chants relativement courts, dépassant rarement les cinq minutes. Il existe des anen pour faciliter la réalisation de l'ensemble des tâches et désirs quotidiens, de la chasse à la pêche en passant par l'horticulture, la séduction amoureuse ou encore l'abattage d'un arbre. Dans son livre consacré à la magie des Aguaruna (Awajún), Brown (1985, p. 133-161) attribue un rôle central aux chants anen qu'il définit comme une véritable « technologie des sentiments » permettant de manipuler secrètement les émotions des autres. Taylor et Chau (1983) s'intéressent au contenu des chants anent ${ }^{3}$ achuar et remarquent une association stable entre les choses et qualités évoquées dans ces incantations et certains sentiments. Taylor (2000) prolonge cette idée en précisant que ces chants magiques décrivent des relations intersubjectives censées mettre en place des affects spécifiques entre le chanteur et le destinataire. Les anent achuar sont des « manifestations de l'affectivité » (Descola 1993a, p. 97), mais leur performativité, postulée dans les discours indigènes, est un point qui reste encore à éclaircir. Pourquoi les Wampis pensent-ils que les anen sont efficaces? Dans cet article, je propose de me focaliser sur l'expérience intime du chasseur énonçant ces chants et de suivre l'intuition de Brown (1984, p. 554) selon laquelle les anen agissent en « créant des émotions » et « en structurant des expériences sensibles ». Pour saisir de quelle manière ces charmes agissent, il faut les inscrire dans un travail corporel qui déborde le seul cadre de leur énonciation. C'est ce que soulignent Amadio (1988), Mader (1999) et surtout Surrallés (2003) en mettant en avant le rôle de la souffrance et du jeûne qui doivent précéder la récitation mentale et l'activité cynégétique. Les effets des anen dépendent de la capacité du chanteur à fournir l'effort nécessaire à la satisfaction des conditions corporelles de leur énonciation rituelle. Ils seront d'autant plus intenses que le « chanteur », l'anentrin (anentrin / anenta-ru-inu / chanter.un.anen-APPL-NZR) est kakaram (kakaram / kaka-rama / être.fortADJECTIVISATEUR), fort, robuste, valeureux, c'est-à-dire capable, entre autres, d'endurer la souffrance qu'il s'inflige ${ }^{4}$.

En suivant les étapes d'une partie de chasse jusqu'au contact avec la proie, je voudrais éclairer ce travail sur soi-même que réalise le chasseur pour parvenir

3. Chez les Shuar et les Achuar, ces incantations sont appelées anent.

4. De manière générale, comme chez les Shuar équatoriens, on peut repérer chez les Wampis une idéologie du langage selon laquelle la performativité de la parole dépend de la force personnelle du locuteur (Gnerre 2009). 
à ses fins. Je me concentrerai sur deux points. Tout d'abord, les deux premières parties s'intéressent aux dispositions corporelles, en particulier celle du « ventre vide » accompagnant le jeûne, qui précèdent la chasse, la transmission et l'énonciation des anen. Ce sera l'occasion d'étudier la manière dont les Wampis exercent un contrôle sur les chants et sur leur ressenti. Ensuite, les troisième et quatrième parties examinent le processus par lequel le chanteur parvient à expérimenter physiquement les comportements et les relations entre humains et non-humains évoqués dans le texte de l'anen. Au fond, le simple fait de « penser » (anentaimat / anentaima-ta / penser-NZR ${ }^{5}$ ) au gibier, ainsi que s'est efforcé de le faire Felipe après son rêve, permet selon lui de visualiser, comme lors de l'énonciation ritualisée des anen mais avec une intensité moindre, la relation que le chasseur veut établir avec l'animal. L'action de l'anen sur son destinataire dépend des affects ressentis par le chanteur: selon les Wampis, si le chanteur fait l'expérience sensible de la relation avec l'allocutaire décrite dans l'incantation alors ce dernier l'éprouvera également. Par une analyse pragmatique et sémantique, je tâcherai de montrer comment les anen contribuent à façonner chez le chanteur des affects ${ }^{6}$ créés intentionnellement.

5. Dans la suite de l'article, tous les verbes wampis cités dans le corps du texte apparaîtront sous leur forme infinitive, c'est-à-dire accompagnés du suffixe nominalisateur -ta qui crée un nom désignant l'action du verbe.

6. Nous savons que les définitions des concepts d' « affect » et d' « émotion » sont extrêmement compliquées à formuler en sciences humaines. Ces dernières années, des auteurs, surtout anglo-saxons et s'inscrivant dans ce qui est désormais appelé " the affective turn " (Massumi 2002; Thrift 2008), ont opposé les deux notions en affirmant que les affects sont des sensations qui ne sont pas médiatisées, contrairement aux émotions, par des processus cognitifs. Pour ma part, j'adopterai la position de Rosaldo (1984) selon laquelle la pensée (les représentations, les cognitions) et la vie affective n'existent pas séparément et s'influencent en permanence réciproquement. La limite entre affect et émotion est par conséquent très floue et ne constitue pas un cadre d'analyse pertinent pour l'étude que je souhaite engager. Dans la suite de l'article, je ne ferai pas de différence conceptuelle entre affect et émotion. Cependant, j'emploierai essentiellement le terme « affect » qui présente l'avantage d'être anthropologiquement plus facile à manipuler que celui d' "émotion » car il évite de circonscrire un ensemble de ressentis à une catégorie essentialisante et réductrice (joie, colère, tristesse, etc.). L'usage du terme « émotion » trahirait la formidable complexité des descriptions des relations entre le chanteur et l'allocutaire présentes dans les anen. Comme nous le verrons, les innombrables « dispositifs linguistiques » qui servent à encoder les aspects affectifs des relations décrites dans les anen (" affect-encoding devices ») (Besnier 1990), témoignent de cette complexité. L'usage rituel de ces chants magiques pose ainsi la question des rapports entre langage et affect. Il ne s'agit plus seulement d'étudier la manière dont les affects sont exprimés et canalisés au travers de codes discursifs comme chez les contributeurs de l'ouvrage collectif d'Abu-Lughod et Lutz (1990), mais de s'intéresser à la dynamique inverse: pour les tenants de cette anthropologie des émotions (Lutz et White 1986), le ressentir précède l'expression, l'interaction sociale et l'utilisation de conventions; ici, c'est l'imbrication des formes discursives et de l'action rituelle qui génère le ressentir. 


\section{Une condition de réussite du chant et de la chasse: « le ventre vide »}

Les chants de chasse wampis ne sont pas désignés par un terme particulier sinon celui de la catégorie générale d'anen par opposition aux chants de fête nampet, ou aux quasi disparus chants ujaj consacrés à la fête de la tsantsa ${ }^{7}$. Seuls les anen sont réputés avoir une influence sur leurs destinataires. Les nampet relatent des évènements de la vie quotidienne comme les jeux difficiles de la vie amoureuse, et les ujaj ceux de la guerre. Les destinataires des anen de chasse wampis peuvent être humains, animaux, végétaux ou artefacts.

Quand l'envie ou la nécessité de gibier se font sentir afin de changer du poisson, des œufs ou des seules bananes vertes bouillies, l'homme se lève tôt, un peu avant le soleil, pour aller chasser. L'expédition se prépare dès la veille. On négocie souvent des cartouches avec le frère, le beau-frère ou le beau-père qui se voient assurés en cas de succès d'obtenir une belle pièce de l'animal. Entre la maisonnée à satisfaire et les créanciers alliés à rembourser, les enjeux de la chasse deviennent vite conséquents. Dès lors, il faut parvenir à obtenir la veille un rêve de chasse de bon augure kuntukmamu ${ }^{8}$ afin de s'assurer de rencontrer du gibier le lendemain (dans l'idéal tayassu pecari, le pécari à lèvres blanches). En l'absence de ce type de rêve, la tâche cynégétique s'annonce délicate. La chasse est une activité de plus en plus difficile aujourd'hui. Dans les grandes communautés (à partir d'environ 800 habitants), elle est devenue une activité secondaire. Les Wampis se consacrent majoritairement à la pêche ou l'élevage de volailles pour subvenir à leurs besoins de consommation animale. Surtout du côté du río Santiago, où se développent depuis peu des programmes nationaux de développement de l'agriculture et de la pisciculture. Quoi qu'il en soit, des deux côtés de la chaîne des collines de Kampankis qui s'étendent du Nord au Sud du territoire wampis, il est devenu compliqué de chasser : l'organisation de la vie en communauté, qui a remplacé une distribution éparse des maisons sur le territoire et la démocratisation du fusil ont eu pour conséquences l'éloignement et la raréfaction croissante du gibier. Dorénavant les chasseurs doivent effectuer des expéditions de plusieurs kilomètres. Malgré tout, la chasse reste une activité hautement valorisée. Les bons chasseurs sont considérés avec respect et les rumeurs de chasse fructueuse se propagent à toute vitesse dans les communautés, ce qui ne manque pas de flatter l'ego de ceux qu'on qualifiera pour l'occasion de kakaram. Le gros gibier représente de plus un capital non négligeable dont la vente peut aider une famille à acheter d'autres produits alimentaires ou de

7. Il s'agit du rituel de la tête réduite ou tsantsa, qui a rendu si célèbres les populations jivaros. Le rituel n'étant plus pratiqué chez les Wampis, les chants ujaj disparaissent des mémoires. J'ai pu cependant en recueillir quelques-uns auprès des femmes les plus âgées.

8. Kuntukmamu est étymologiquement apparenté à kuntina, « animal » ou " gibier », kuntut, « être gros », et kuntutin qui désigne l'époque de l'année où les animaux s'engraissent (entre décembre et mai). 
l'essence. Lorsque je réalisais mon enquête ethnographique, le kilo de gibier se négociait entre 6 et 8 Nuevos Soles (entre 1,74 et 2,30€), voire à $10 \mathrm{~S} \backslash$. $(2,89 €)$ pour la viande de paca (Cuniculus paca) très tendre et savoureuse.

Ainsi, le chasseur assidu et attentif aux techniques de chasse transmises par ses aïeux partira à la chasse à l'aube, accompagné ou non de son chien, l'estomac uniquement rempli d'un ou deux bols de nijamanch, la bière de manioc. Il emportera avec lui son fusil et beaucoup plus rarement sa sarbacane. La sarbacane et les fléchettes au curare sont utilisées pour chasser les oiseaux ou les singes mais seulement par le chasseur averti, celui qui aime la discrétion féline du bon prédateur à l'approche de sa proie. Discrétion faisant défaut avec le fusil si par malheur l'homme manque son tir. Partir à la chasse le ventre vide est une condition de réussite cynégétique importante: c'est une application du principe de la « souffrance » (waitamu / waita-mau / souffrir-NZR) que l'on retrouve dans de nombreuses sphères de la vie quotidienne ou rituelle wampis 9 . Une certaine retenue alimentaire est également de mise si l'on veut que les anen soient efficaces. Pourquoi partir chasser le ventre vide? Les Wampis « souffrent » (waitut / waitu-ta / souffrir-NZR) quand ils ont « l'estomac mort/ blessé » (wake mesemar), un état que les informateurs traduisent en espagnol tantôt par « être triste », tantôt par « avoir le ventre vide ». Avoir le ventre vide signifie plusieurs choses: que l'on n'est pas assez fort pour pouvoir se nourrir seul, et que personne n'est là pour vous prendre en pitié et vous donner de quoi vous alimenter. Or l'ethnographie des sociétés jivaro et mon expérience de terrain ont montré qu'être fort, et être perçu comme tel par les autres membres de la communauté, c'est être capable de contracter des alliances, au sens matrimonial ou au sens politique, que ce soit avec des Wampis, des étrangers ou des non-humains. Un homme se doit de trouver une femme et une belle-famille qui veuillent bien l'intégrer à leur groupe pour se réaliser individuellement. Il est intéressant à cet égard de constater que l'adjectif natsa signifie à la fois « jeune » et « célibataire ». Un homme sans femme ne pourra pas se socialiser ni créer d'autres alliances car la sociabilité wampis n'est pas possible sans la bière de manioc, confectionnée exclusivement par les femmes. Il est inimaginable d'inviter quelqu'un dans sa demeure sans lui servir un bol de cette boisson laiteuse. Une maisonnée bien tenue en regorge: il y a toujours quelque chose pour se remplir le ventre. Celui qui a le ventre vide donc, ou bien est un homme faible, ou bien est un enfant (abandonné), deux propositions quasiment équivalentes chez les Wampis. Être triste, souffrir, « avoir le ventre

9. C'est le cas par exemple lors de la recherche des esprits puissants arutam avec jeûne et prise de psychotropes, pendant laquelle hommes et femmes cherchent à acquérir l'assurance d'un futur radieux, rituel caractéristique de l'ensemble linguistique et culturel jivaro, incluant les Candoshi et les Shapra (Harner 1972; Pellizzaro 1990; Descola 1986, 1993; Taylor 1997, 2003 ; Mader 1999; Surrallés 2003; Boster 2003 ; la figure d'arutam est évoquée très brièvement par Seymour-Smith 1988, p. 217 pour le cas Shiwiar). 
vide », c'est ne pas avoir d'alliés, se sentir seul. Or, lorsqu'on voit un individu au « ventre vide », on « éprouve de la compassion » (wait anentrut) ${ }^{10}$ pour lui, ce qui se traduit par un don de nourriture. L'individu faible, triste, esseulé et affamé se trouve contraint de susciter de la compassion chez celui qui peut le nourrir, et donc d'accepter une relation de dépendance. Cette dynamique est généralisée à toute instauration d'une nouvelle relation d'alliance, à l'initiative de la personne souffrante. Celui qui compatit ne donne pas forcément de la nourriture mais peut aussi communiquer de la force ou un savoir. L'exhibition de la souffrance du « ventre vide » a donc pour effet de pousser l'autre à agir en faveur de celui qui souffre. La souffrance peut être ainsi volontaire et servir à créer une relation de dépendance afin de satisfaire le « ventre vide », de sorte qu'être « fort » (kakaram) nécessite d'en passer par un moment de souffrance et de dépendance.

Par ailleurs, avoir le « ventre vide » et donc « avoir faim » (tsukamat / tsukama-ta / faim-VZR-NZR) est une des motivations pour aller chasser et devenir bon chasseur, c'est-à-dire un homme que l'on qualifie en wampis de uwetkartinchau (uwetut-karatu-inu-chau, ne.pas.trouver-1PL.OBJ-NZR-NÉGATION, « celui qui ne manque pas de nous trouver [du gibier] »). Hommes et femmes wampis se plaignent rarement et ne montrent pas qu'ils sont tenaillés par la faim quand la situation ne permet pas de se restaurer. C'était le cas pendant les longs voyages en pirogue à contre-courant, où nous restions assis patiemment, bercés par le bruit du moteur, « le ventre vide », pendant neuf ou dix heures. Voyant que je m'agitais sur mon siège formé d'une planche de bois pour essayer de faire affluer le sang dans mes membres endoloris, ils prenaient plaisir à me raconter leurs plus dures épopées sur le fleuve: sans manger, de nuit et sous la pluie. Pour faire mon éducation à l'éthique wampis de la souffrance, ils me répétaient fièrement l'invariable leçon à la fin de leurs histoires : " hay que aguantar », « il faut résister ». Nous aurions pu prévoir d'apporter de la nourriture ou même de débarquer quelques minutes dans l'une des communautés qui bordaient notre route, mais mes amis avaient cette volonté de se mettre à l'épreuve - comme lors de la prise rituelle du psychotrope Banisteriosis caapi-pour montrer qu'ils étaient « forts » (kakaram). La vie quotidienne wampis est parsemée de ces occasions offrant la possibilité de démontrer publiquement son endurance

10. On ne peut manquer de faire le rapprochement entre anentrut / anenta-ru-ta / chanter. un.anen-APPL-NZR et wait anentrut, " éprouver de la compassion ». Le morphème wait, lorsqu'il est seul, est un verbe signifiant « entrer dans » ou "pénétrer ». Comme nous le verrons un peu plus loin, pour les Wampis, chanter un anen permet de manipuler le « cœur» (anentai), siège des pensées et des émotions du destinataire. L'hypothèse que nous pouvons formuler est que si « chanter un anen » permet au sujet chantant de manipuler le destinataire en se plaçant, entre autres, dans une position de personne souffrante, wait anentrut désigne cette action de manipulation mais du point de vue du destinataire manipulé qui est alors le sujet du verbe. Autrement dit, pour les Wampis, si le destinataire éprouve de la compassion, cela signifie qu'il « s'est laissé pénétrer » et manipuler le " cœur » par celui qui souffre. 
et sa force personnelle face à la faim, la fatigue et la souffrance, surtout dans le cadre des activités masculines. Au-delà de l'aide qu'ils peuvent s'apporter mutuellement, les hommes instaurent quasi inévitablement un climat de compétition, ouvertement déclarée ou non, durant l'essartage, la construction d'une maison, ou les longs débats politiques dans la salle communale. Les honneurs reviendront à ceux qui auront le plus travaillé et le moins mangé. Endurer le « ventre vide » n'est pas seulement un moyen d'attirer la compassion d'autrui mais aussi de susciter son admiration.

Il apparaît maintenant beaucoup plus clairement pourquoi il est recommandé au chasseur wampis de partir en forêt sans manger. Le schème du « ventre vide » permet de constituer un positionnement qui rend possible simultanément deux types de relations différentes: une relation de dépendance par génération de tristesse et de compassion et une relation de prédation causée par la sensation de faim. La première va permettre au chasseur de pousser les protagonistes de l'interaction cynégétique - proie incluse - à s'engager en sa faveur, tandis que la deuxième va motiver l'homme à réaliser l'action prédatrice et à devenir un « bon chasseur » uwetkartinchau. C'est là qu'interviennent les chants magiques anen qui nous occupent. Ils auront pour but d'instaurer l'un et/ou l'autre de ces deux types de relations avec le gibier. Le jeûne est donc d'une importance capitale pour notre analyse de la pragmatique des anen et perçu comme essentiel à leur efficacité. Nous commençons donc à nous apercevoir que les dispositions affectives sont utilisées ici comme des outils que le chasseur wampis manie à des fins tout à fait pratiques: attirer, mettre à mort et manger un animal.

\section{L'apprentissage et l'énonciation des anen : devenir un anentrin}

Continuons notre exploration et regardons à quels moments se chantent les anen. J'ai pu en distinguer de deux types: ceux énoncés de manière répétitive en guise de préparatifs dans la communauté ou en marchant dans la forêt, et ceux énoncés de manière très ponctuelle au moment du tir, pas plus d'une ou deux itérations. Dans les deux cas, l'énonciation du chant est toujours silencieuse ou murmurée de manière à s'assurer qu'il soit tout à fait inaudible pour le destinataire. L'allocutaire ne doit pas savoir qu'une personne tente de l'influencer par un chant magique. Chanter un anen sotto voce se traduit en wampis par iñitiak anentrut. Anentrut / anenta-ru-ta / chanter.un.anen-APPL-NZR est l'action de chanter l'anen à quelqu'un et iñitiak un adverbe signifiant « à l'intérieur », « profondément ». Les jivarologues ont remarqué le lien étymologique existant entre le mot anen et le mot cœur anentai ${ }^{11}$ (Taylor et Chau 1983; Descola 1986, p. 246; Surrallés 2003, p. 227) ce qui a valu aux chants d'être

11. Anentai ou enentai pour les Awajún et Wampis, ininti pour les Achuar. Mais cela n'enlève ou ne rajoute rien à la signification du mot. 
nommés « discours du cœur» par Descola (1993a, p. 219). Selon les Wampis, les anen ont pour but de pénétrer directement le cœur pour influencer les pensées et les sentiments du destinataire. Comme les Candoshi (Surrallés 2003, p. 62-73) et les Achuar (Descola 1986, p. 253), les Wampis considèrent le cœur comme étant à la fois le siège des émotions et de la cognition ${ }^{12}$. Dans le corpus que j'ai recueilli, les chants de chasse ne s'adressent pas à un « maître » du gibier (Reichel-Dolmatoff 1973; Chaumeil 2010; Gutierrez Choquevilca 2012), ni au chef ou au représentant le plus grand de l'espèce chassée (Descola 1986), mais directement à un individu lambda afin d'influer sur son comportement. Inspiré par les textes de Chaumeil (1993) et Gutierrez Choquevilca (2012), je dirais même qu'il s'agit pour le chanteur, dans le cas de certains anen, de momentanément substituer sa voix à celle de sa proie. Si le gibier entendait le locuteur chanter, il se mettrait sur ses gardes et prendrait sûrement la fuite. Mais cette subvocalisation n'est pas seulement une mesure de discrétion, elle confère au chant son pouvoir et rend ses paroles performatives. Voyons pourquoi.

On se souvient que l'homme wampis, la veille de partir dans une expédition cynégétique, va tenter d'avoir un rêve de bon augure kuntukmamu, qui assurera son succès. Pour certains, c'est même une condition nécessaire pour aller chasser. À défaut, ils se consacreront à une autre activité en attendant de tenter à nouveau leur chance la nuit suivante. Les évènements qui se déroulent dans les rêves ou les visions induites par l'ingestion de psychotropes sont pour les Wampis des expériences pertinentes, à interpréter ou à prendre littéralement, pour prendre des décisions et agir dans la vie éveillée ${ }^{13}$. Comme pour leurs voisins et familiers Shuar d'Équateur (Rubenstein 2012), les Wampis considèrent que ces expériences oniriques et visionnaires sont même plus fiables que celles vécues en état de veille. Pour celui qui sait les comprendre, elles ne « trompent » (tsanumat / tsanu-ma-ta / mensonge-VZR-NZR) ${ }^{14}$ jamais. La trame archétypale du rêve kuntukmamu consiste à rencontrer un homme ayant un comportement belliqueux envers le rêveur. Cette personne est interprétée comme étant l'animal que le chasseur poursuivra à l'aube. J'avais soutenu ailleurs que ces rêves constituaient pour les chasseurs Jivaro l'assurance d'une amorce de relation avec l'animal qu'il faudrait achever une fois éveillé par la mise à mort

12. Des analyses linguistiques confirment ces remarques. Anentai est probablement dérivé de anee-, « aimer » auquel s'ajoute le suffixe nominalisateur -tai, donnant le sens de " ce avec quoi on aime ». Le verbe anentaimat, " penser », se décompose en anentaimat / anentai-ma-ta / cœur-VZR-NZR: « penser » et « aimer » sont des activités du cœur.

13. Je renvoie ici aux articles de Descola (1989) pour l'interprétation des rêves chez les Achuar ou à l'article d'Yvinec (2005) traitant plus largement des rapports aux non-humains en Amazonie, notamment à travers les rêves.

14. Tsanumat a le sens de « duper», « induire en erreur » ou encore « leurrer ». 
du gibier ${ }^{15}$. Comme dans les récits mythiques qui décrivent un monde révolu, les animaux peuvent apparaître dans les rêves sous forme humaine, contexte communicationnel dans lequel les deux interlocuteurs se comprennent parfaitement. Les rêves wampis sont le lieu où les wakan, " esprits » des humains ou des non-humains, s'entendent et interagissent, une telle communication étant bien évidemment impossible dans la vie éveillée. Chaque nouveau chant magique anen est enseigné aux Wampis au cours d'une expérience onirique ou visionnaire (les chants ont été à l'origine « rêvés », karamaya / kara-ma-ya / rêve-VZR-PASSÉ.LOINTAIN). Selon les individus réputés connaître de nombreux chants magiques, il n'est pas possible d'inventer soi-même un anen $^{16}$. Pour être efficient il doit toujours, à l'origine (avant de circuler entre humains), être transmis par un esprit arutam, qui peut se présenter sous les traits d'un ancêtre wampis ou de n'importe quel animal ${ }^{17}$. En énonçant l'anen, en usant de cette forme discursive d'origine onirique compréhensible à la fois par les humains et les non-humains, le chasseur tenterait ainsi de continuer la relation débutée avec le gibier dans le rêve kuntukmamu.

En énonçant silencieusement les anen, les Wampis introduisent dans les activités de la vie éveillée une modalité d'échange avec les non-humains propre à l'espace onirique où la communication est parfaitement possible entre wakan, « esprits ». Pour les Wampis, les paroles transmises dans les rêves/visions ou les récits des évènements qui s'y déroulent possèdent une force permettant d'exercer une influence sur les évènements de la vie éveillée. Du temps où régnait un climat de vendetta généralisée entre groupes d'alliés ${ }^{18}$ ou aujourd'hui lorsque sont organisées des manifestations pour défendre les territoires communautaires face aux entreprises extractivistes, les « visionnaires » (waimaku / wai-ma-ka-u / voir-RÉFLÉCHI-INTENSIFICATEUR-NZR) transmettent aux jeunes qui s'apprêtent à s'engager dans une expédition potentiellement dangereuse leur rêve de bon augure de la veille annonçant leur victoire. Cet acte est appelé en wampis kara atit / kara ati-ta / rêve détacher-NZR, littéralement « détacher le rêve ». En m'expliquant ce terme, un de mes hôtes a comparé cet acte de parole à celui de détacher une pirogue sur le cours d'un fleuve. En verbalisant et en adressant son rêve à ceux qui partent, le waimaku leur transmet la force que le rêve de bon augure lui avait prodiguée. Au même moment, il perd cette force

15. J'ai défendu cette hypothèse dans mon mémoire de master L'environnement du rêve. Oniromancie et Arutam chez les Jivaro, soutenu à l'École des hautes études en sciences sociales à Paris en 2012.

16. Les chants de fêtes nampet peuvent être inventés.

17. Il peut également apparaître sous la forme d'un phénomène météorologique comme un vent violent ou le tonnerre.

18. Comme chez leurs voisins Achuar, Shuar, Awajún ou Candoshi, un phénomène social amplement documenté et glosé dans la littérature sur les groupes jivaros (voir par exemple Taylor 1985; Descola 1993a, 1993b; Surrallés 2000, 2003; Steel 1999). 
lui-même, comme s'il avait lâché son bateau au gré des flots. Les Wampis disent qu'en entendant le récit du rêve, les destinataires se sentent renforcés, prêts à affronter les éventuels dangers. Le récit onirique semble être alors performatif: les allocutaires, encouragés, partent confiants et déjà victorieux, absolument certains que le contenu du rêve va se réaliser. En délivrant son récit, le waimaku reproduit le contenu affectif de son vécu onirique et de sa rencontre avec l'esprit afin que les allocutaires se le réapproprient, par exemple le courage, la confiance ou un certain enthousiasme guerrier. Il en est de même pour les chants anen, initialement hérités d'un esprit arutam rencontré en songe. Si les anen sont perçus comme performatifs, c'est en partie parce qu'ils renvoient à l'expérience affective initiale du visionnaire lors de sa rencontre avec l'arutam.

Cependant une différence reste notable entre « détacher le rêve » (kara atit) et « chanter un anen » (anentrut) : le visionnaire délivre son rêve à voix haute, le chanteur énonce son anen sotto voce. En faisant cela, du point de vue wampis, le premier perd le pouvoir de son rêve pour le donner à l'allocutaire tandis que le deuxième le conserve, il garde l'anen. Dans le premier cas le destinataire, en entendant le message, devient le possesseur et contrôle la force qu'il porte, dans le second cas, il ne l'entend pas et est manipulé par le chanteur récitant le charme magique. La subvocalisation du chant permet de conserver le contrôle de l'anen. Nous allons voir qu'apprendre un anen, c'est en devenir le «possesseur » et le « maître » pour en contrôler les effets. L'apprenant devient alors un anentrin, un " chanteur », ou, de manière synonymique, un anentrintin / anenta-rin-tinu / chant.anen-3SG.POSS-ATTRIB, « un maître/possesseur de chants anen ». Tentons d'éclaircir dès à présent ce point.

Nous le savons depuis Harner (1972) pour les Shuar et Brown (1985) pour les Awajún (ou Aguaruna), la transmission des anen s'effectue selon la division sexuelle du travail. Les chants de chasse sont transmis de pères en fils, les chants d'horticulture de mères en filles. Le mode opératoire, décrit chez Brown, reste à peu près le même chez les Wampis. Le maître commence par fumer du tabac, puis souffle la fumée directement dans la bouche de son élève à l'aide d'une paille de bambou. L'opération est renouvelée trois ou quatre fois de manière à ce que les effets de la nicotine ${ }^{19}$ se fassent sentir. Ensuite, le père communique l'anen à son fils: une première fois chanté, une deuxième fois parlé. Il peut réitérer la chose en fonction des capacités d'apprentissage du jeune homme. À chaque fois que le père termine une récitation, l'élève doit avaler sa salive. Selon le beau-père d'un de mes amis de Chinganaza, une communauté de la rive occidentale du río Santiago, avaler sa propre salive après chaque énonciation est le secret d'un apprentissage réussi. Chez les Awajún, il existe même un échange de salive et de jus de tabac entre le maître et l'élève (Brown 1985,

19. Le tabac utilisé en Amazonie est l'espèce Nicotiana rustica, dont la teneur en nicotine est jusqu'à dix fois supérieure à celle du tabac commun Nicotiana tabacum. 
p. 72). La salive semble être perçue par les Wampis comme le réceptacle de l'anen transmis. En associant le chant avec la salive, la déglutition sera le moyen d'incorporer l'anen "profondément ", «à l'intérieur 》 (iñitiak) afin de le posséder et de le maitriser. L'analogie ne peut pas manquer d'être faite avec les tsentsak, les fléchettes magiques des apprentis chamanes uwishin qu'ils reçoivent de la part d'un autre chamane sous la forme d'un flegme salivaire et qu'ils gardent dans leur estomac. Nous comprenons mieux pourquoi il faut jeûner avant de partir à la chasse et d'énoncer des anen: l'estomac est le lieu de création et d'incorporation par excellence des relations (par la nourriture et la commensalité), et des différents pouvoirs anen et tsentsak.

La transmission des anen entre humains est portée par une conception physiologique de l'apprentissage comme acte de nourrissement ${ }^{20}$. Cet acte sous-tend la production (et reproduction) d'une relation de dépendance de type enfantsparents que l'on retrouve lors de l'acquisition première des anen dans un contexte de rencontre avec un arutam. Durant la session rituelle de prise de psychotrope le participant adoptera, grâce à un autre anen facilitant l'apparition de l'esprit puissant et au « ventre vide », une position d'enfant abandonné demandant à son " grand-père » de lui communiquer un nouveau chant. Suite à cette acquisition première, le parent transmettra l'anen selon le procédé que l'on vient de détailler en donnant à son enfant, stricto sensu, le chant magique à « ingérer ». Le chanteur d'anen, celui qui connait et possède des chants magiques, se nomme l'anentrintin / anenta-rin-tinu / chant.anen-3sG.POSS-ATTRIB. La suffixation au substantif anen du possessif -rin suivi de l'attributif -tinu, traduit une relation de possession (Overall 2007, p. 208-209; Peña 2015, p. 475-477) qui selon mon analyse implique l'idée de contrôle ${ }^{21}$ ou de maitrise ${ }^{22}$. Outre le terme waimaku qui désigne celui qui a une vision d'un arutam, les Wampis utilisent également le mot karintin / kara-rin-tinu / rêve-3SG.POSS-ATTRIB, « celui qui possède un rêve, une vision ». Et comme nous l'avons vu plus haut, celui qui possède un rêve (sous-entendu de bon augure), contrôle ce rêve car il peut choisir d'en faire le récit et donc de le « délivrer », ou de le garder pour lui. La possession impliquant l'idée de contrôle est manifeste dans le mot jeentin / jea-rin-tinu / maison-3sG.POSs-ATTRIB que les Wampis traduisent en espagnol par « dueño de la casa », le «maître de maison ». C'est celui à qui appartient l'habitation,

20. Je préfère employer le mot «nourrissement»-usité en français dans un cadre particulier par les apiculteurs - plutôt qu' « acte alimentaire » car le premier exprime mieux la relation de dépendance entre celui qui nourrit et celui qui se nourrit alors qu'elle peut être absente dans le second, puisqu'il est possible de se nourrir seul.

21. Gutierrez Choquevilca (2013, p. 39) remarque que les Quechua du Pastaza usent également du « schème de la commensalité » ou de l'argument du «nourrissage » dans un chant adressé aux hordes de pécaris à lèvres blanches pour les influencer et les contrôler.

22. Le thème de la maîtrise est devenu de première importance en anthropologie amazoniste avec entre autres l'article de Fausto (2008). 
un homme, et qui en contrôle la construction, l'entretien ainsi que les allées et venues des hôtes adultes masculins. L'anentrintin (ou anentrin) est un homme qui détient mais surtout sait contrôler ces charmes magiques en respectant l'ensemble du dispositif rituel qui leur confère leur efficacité, à commencer par le « ventre vide ». Lors de l'apprentissage, la relation de possession et de contrôle que l'élève aura avec le chant est concrétisée par 1'acte de nourrissement ${ }^{23}$.

Le chasseur wampis désire avant tout créer une relation de prédation avec le gibier. Pour ce faire, il associe l'anen au souffle et à un projectile de sarbacane. Cette association est réalisée très clairement dans le geste de clôture de l'énonciation du chant. Mader remarque ce geste chez les Shuar et les Achuar mais ne le commente pas beaucoup (Mader 1999, p. 228, 270). Quand les Wampis veulent influencer des individus qu'ils estiment puissants, ils passent une main devant leur bouche à la fin de l'anen ou d'une parole qu'ils veulent performative en prononçant l'onomatopée « kusui! » qui évoque le son d'un tir à la sarbacane. La main passe rapidement au moment de dire la deuxième syllabe -sui, coupant un instant le souffle naturel de l'énonciation du mot. Le geste donne l'impression sonore d'une fléchette lancée avec une sarbacane. Les chamanes uwishin utilisent la même onomatopée lors du lancer de leur tsentsak pour nuire à quelqu'un. Pour le chasseur qui énonce un anen cynégétique, ponctuer le chant par ce son équivaut à lancer un sort vers sa proie. Si le chasseur estime que la proie n'est pas très puissante, il peut très bien s'abstenir de ce geste final. Le « kusui » n'est donc pas une condition nécessaire au fonctionnement du chant. Cependant, il permet d'accroître le contrôle de l'anentrin sur l'anen en lui donnant l'occasion d'intervenir sur plusieurs paramètres : il peut donner une direction au lancé (vers l'allocutaire), moduler l'intensité sonore de la voix au moment de dire " kusui », ou encore exécuter un geste de la main passant devant la bouche plus ou moins sec et brusque. Pour les Wampis, ce contrôle permet de conférer une force de pénétration plus importante au chant, à la manière d'une fléchette, pour atteindre le cœur des destinataires et remplacer discrètement leur voix par celle du chanteur ${ }^{24}$. Cette onomatopée est d'ailleurs utilisée dans la vie de tous les jours. Par exemple, elle est prononcée de bon matin pour inciter le soleil à se lever. Les Wampis prennent de la cendre dans le foyer puis la jettent devant eux au niveau de leur tête en disant "Kusui! Etsachi etsantrata! », " Kusui! Petit soleil brille! » (etsachi etsantrata / etsa-chi etsantra-ta / soleil-DIMINUTIF briller-IMPÉRATIF.2SG). Si le nuage de cendre tombe tout de suite à terre, le ciel s'annonce couvert, s'il monte et reste en suspension un petit moment, il fera

23. On trouve, entre l'apprenti et l'anen ingéré, un rapport contenant-contenu, schème amazonien caractéristique des relations de maîtrise et de contrôle (Fausto 2008; Costa 2010).

24. En ce sens, Surrallés remarque que les Candoshi «n'établissent pas de différence substantielle $[\ldots]$ entre une fléchette de sarbacane et une incantation, aussi bien dans leurs capacités concrètes que dans la nature de leur ontologie » (2003, p. 229). 
beau. Il semble que la cendre suive le mouvement du soleil levant qui, sous l'effet du kusui, s'est plantée dans son corps, comme une fléchette de sarbacane dans un animal. Quand le nuage de cendre chute immédiatement, cela signifie que la cible a réussi à esquiver le projectile, un acte guère étonnant de la part d'un être mythique aussi puissant qu'Etsa, le soleil ${ }^{25}$.

Les associations du chant à la salive et à une fléchette permettent non seulement au chanteur de contrôler l'anen mais également de faire l'expérience sensible, avec les deux gestes caractéristiques de l'acte de nourrissement et du lancer " kusui », des deux grands types de relations que le chant est censé générer et que le chanteur devra maîtriser pour parvenir à ses fins : la relation de prédation et la relation de dépendance. Dans ce contexte cynégétique, on verra que cette dernière relation qui, rappelons-le, est amorcée par la disposition du « ventre vide », pourra être utilisée à la manière d'un leurre affectif.

\section{Générer des relations affectives pour réussir la chasse}

Les deux premières parties nous ont permis de comprendre le contexte et les conditions pragmatiques générales de l'énonciation des anen. Je vais maintenant étudier le contenu textuel de ces chants. Un chasseur wampis aguerri a bien voulu m'expliquer de quelle manière il faut énoncer le chant. L'anen ne fonctionne que lorsque l'on « pense bien », shir anentaimat (shir anentaimat ) shir anentaima-ta / bien penser-NZR) (c'est-à-dire en concentrant son attention) à la scène qu'il décrit et aux destinataires auxquels il est adressé. La récitation de l'anen peut avoir comme but d'agir sur le chanteur lui-même, pour devenir un bon chasseur par exemple, et/ou sur le gibier afin de l'attirer. Un chant énoncé à voix haute sans effort de ce type n'aurait aucun impact. Dès que la scène évoquée dans l'anen est bien pensée, les Wampis disent qu'elle se réalise. Suivant cette logique, le destinataire pense et agit alors comme indiqué dans le chant et le succès de l'expédition cynégétique est assuré.

La méthode que je mets en œuvre pour étudier les chants et leur usage par les chasseurs consiste en un aller-retour entre deux niveaux d'analyse. Le premier est centré sur le texte des anen. Je relève les dispositifs linguistiques qui permettent d'encoder les aspects corporels et affectifs de l'attitude que le chanteur veut adopter et de la relation qu'il veut établir avec les allocutaires. Ce niveau d'analyse rend compte de la complexité sémantique du texte en s'appuyant sur les éléments morphosyntaxiques importants et en révélant les dimensions mythiques, écologiques, sociologiques et praxéologiques auxquelles le texte fait référence. Au second niveau, j'inscris l'anen dans le contexte cynégétique de son énonciation. Par là, j’essaie de saisir les conséquences de

25. C'est Etsa, le soleil, qui enseigna aux Wampis les techniques de chasse et de fabrication de la sarbacane. 
l'effort shir anentaimat sur le ressenti des anentrin en me fondant pour une grande part sur leurs explications des effets de cette subvocalisation rituelle. Je pars du principe, dans cet article, que le sens du texte est limpide pour le chanteur. En décrivant l'expérience sensible de l'anentrin récitant l'anen, on contribue ainsi à l'élucidation de l'idée de la qualité performative des chants magiques puisque pour les Wampis, ressentir la relation avec l'allocutaire décrite dans l'incantation équivaut à la réaliser. Par cette méthode, je poursuis l'ambition de mieux saisir comment le chant magique influence la pratique cynégétique. Analysons tout de suite un premier chant.

Au vers 1, le chanteur commence par s'identifier au tsemna pinchu (Leptodon cayanensis), une espèce de milan, un des archétypes du bon chasseur qui « nous trouve du gibier », uwetkartinchau. Il s'agit d'un anen de préparation qui s'énonce au moment de s'enfoncer dans l'épaisse végétation. Le chasseur se met en condition pour générer des attitudes corporelles et des comportements propices à la réussite cynégétique en invoquant l'image du rapace et ses qualités prédatrices. L'anen décrit par hypotypose sa future relation avec la proie. Aux vers 3 et 4, le verbe wajan-ta-ja-i / se mettre debout-FUTUR. IMMÉDIAT-1SG-DECL, marque que l'action de «tenir sa proie entre ses serres » comme le fait le milan, va se réaliser dans un futur très proche. L'hypotypose continue dans la suite du chant. Le chanteur possède le même comportement que le rapace: il peut « enserrer » sa proie avec le verbe nominalisé namur$k u$-ta-keku / enserrer-SIMULTANÉITÉ-GÉRONDIF-EXPL ${ }^{26}$, et devient même nyctalope puisqu'il est capable de pousser « en pleine obscurité », un potentiel concurrent de chasse vers le serpent. Comme le chanteur, il faut bien imaginer la scène: le chasseur se met en route quand le soleil commence tout juste à se lever et que le ciel est « jaunissant ». Dans les coins de la forêt où se cachent les serpents, l'obscurité règne encore mais le chasseur est capable de les voir et d'y pousser un concurrent qui traquerait la même proie que lui. Le mot imianu au vers 9 , 10 et 15 , que je traduis ici par « habilement », atteste que cet autre chasseur a obtenu comme lui une vision sous l'emprise de psychotropes lui permettant de maîtriser l'ensemble des techniques nécessaires à la réussite des tâches quotidiennes ${ }^{27}$. L'individu qui a eu ce type de visions est un homme fort et un

26. -keku sont très certainement des syllabes ornementales prononcées uniquement par ce chanteur. Je ne les rencontre que dans les chants de cet anentrin.

27. Les Wampis avec lesquels j'ai travaillé les traductions emploient le terme mélioratif imianu pour qualifier les actions de ceux qui ont obtenu une vision et/ou qui agissent conformément au principe du buen vivir, « bien vivre », tarimat pujut (littéralement " vivre droitement »). Le «bien vivre » est un principe que se sont appropriés les fédérations de communautés wampis et les ONG locales de défense du territoire et de la culture. Il est le leitmotiv de nombreuses luttes indigènes en Amérique Latine, notamment en Équateur et en Bolivie où il est désormais inscrit dans la constitution (depuis 2008 pour l'Equateur, 2009 pour la Bolivie). Il est aujourd'hui la clef de voûte de la lutte politique wampis et 
Anen 1 - Tsemna pinchu (chanteur: Pedro Chuim)

1) Tsemna pinchu asankekuwa $(\times 5)$

2) Kashikikuwa, kashikiku, kashikiku, kashikikuwa

3) Namurkutakeku wajantajai

4) Namurkutake wajantajai, wajantajai, wajantajai

5) Uwetkartichukekuwa

6) Kashikiku yankukiyuku

7) Kashikiku tsemna pinchuwa asankekuwa

8) Kashikikuwa yankukiyaitu, yankukiyaita

9) Chikichaya imianuwa

10) Chikicha imianuwa emkimtaisha

11) Emkimtaishanu napinmakeku emtiki, emtikiajai, emtiki

12) Kashinmakekuwa emtikiajai

13) Kashinmakeku emtikiajai

14) Tsemna pinchuwa asankekuwu, asankekuwa

15) Imianu emkimtaishu napinmakeku emtikiajai, emtikiajai.
Je suis le milan (Leptodon cayanensis)

Tôt, très tôt le matin

Je vais me mettre debout enserrant [la proie]

Je vais me mettre debout enserrant [la proie]

[Je suis celui] qui nous trouve [du gibier]

Tôt le matin, quand le ciel est jaunissant

Tôt le matin, je suis le milan

Tôt le matin quand le ciel est jaunissant

Si un autre, habilement [à la manière d'un bon chasseur ayant obtenu une vision]

Si un autre [bon chasseur] me dépasse habilement

S'il me dépasse, je le pousse vers le serpent

En pleine obscurité, je le pousse [vers le serpent]

En pleine obscurité, je le pousse [vers le serpent]

Je suis le milan

Si on [un autre bon chasseur] me dépasse habilement, je le pousse vers le serpent.

bon chasseur. En se plaçant en concurrence avec un tel personnage, le chanteur sous-entend qu'il est lui-même un visionnaire, avertissant que nul ne saurait se mettre en travers du chemin de sa réussite cynégétique.

Il n'est pas fait mention exacte de l'espèce que le chasseur s'apprête à tuer. La relation de prédation avec la proie n'est qu'à l'état embryonnaire et existe seulement au travers de la présence grandissante du redoutable tsemna pinchu. Cet anen est essentiellement auto-réflexif et vise principalement à faire du chanteur un excellent chasseur. Ainsi, l'acquisition des qualités prédatrices du rapace est amorcée en posant tsemna pinchu en position de prédicat avec la copule

d'une idéologie selon laquelle, entre autres, une conduite en accord avec les pratiques et connaissances « ancestrales », légitimée par un élan écologiste et combinée aux préceptes moraux importés par les missionnaires chrétiens, permettra à la société wampis de survivre aux changements engendrés par la politique économique capitaliste de l'État central péruvien. 
asankekuwa / asa-nu-keku-wa / COPULE-1SG-EXPL-VOCATIF, traduisible par « je suis le Leptodon cayanensis ». Cet anen octroie au chanteur le comportement, les qualités et les motivations prédatrices du tsemna pinchu en lui permettant de conserver la maîtrise de la relation avec la proie et en éliminant tout concurrent. Ainsi aux vers 11, 12, 13 et 15, le chant fait intervenir un cinquième protagoniste (en plus du chanteur, du milan, de la proie et de l'autre chasseur), le serpent. Les Wampis admettent qu'un individu seul ne peut pas échapper à la morsure d'un serpent, sous-entendu venimeux, s'il y a contact avec le reptile ${ }^{28}$. Poussé vers le serpent, l'autre chasseur se retrouvera ainsi forcément en position de faiblesse et perdra son attitude assurée de bon chasseur. Au vers 14, la proposition « je suis le milan » réapparaît pour maintenir la position de contrôle de la relation de prédation face à la menace de ce compétiteur cynégétique.

Avec ce premier anen, le chanteur essaie de se comporter comme le tsemna pinchu, implacable prédateur, ne laissant aucune chance à d'autres concurrents de chasse. L'acquisition de ce comportement est facilitée par l'effort shir anentaimat de visualisation de deux interactions différentes, la première avec le gibier et la seconde avec un autre chasseur, dans lesquelles le chanteur agit comme un milan conservant jalousement sa proie.

L'étape suivante du raid cynégétique est celle du « contact » où le chasseur connaît précisément l'identité de la proie, le nom de l'espèce qu'il souhaite mettre à mort. Le prochain anen s'énonce lorsque le chasseur est à la recherche du singe tseem (Saimiri sciureus ${ }^{29}$ ), proie préférée des rapaces comme l'aigle orné Spizaetus ornatus. Le but du chant est d'apeurer le petit primate afin qu'il se mette à crier. Le cri permet au chasseur de localiser la bête dans la canopée. L'homme possède ici une représentation claire de l'animal qu'il veut chasser. Le singe n'est jamais nommé directement dans l'anen. Cependant, sa présence se manifeste par une forme de discours direct libre par lequel l'anentrin prend possession de la voix de sa proie, manipule ses pensées et lui impose un ressenti.

Jusqu'au vers 5, l'anentrin commence par acquérir les qualités prédatrices du churuwi pinchu. Cette fois-ci, l'identification avec un puissant prédateur se réalise par une auto-nomination directe aux vers 1 et 2 . Au vers 3 et 4, le texte définit 1'espace dans lequel l'aigle évolue, le ciel, avec yaki/haut. Au vers 5, il use de la copule asankekuwa pour s'identifier clairement à l'aigle orné. $\mathrm{Au}$ vers 4 , le terme yanku-ki-ja-i / jaune-DEPLACEMENT-1SG-DECL ${ }^{30}$, littéralement

28. L'unique manière trouvée par les Wampis pour négocier une relation avec les serpents est de réaliser des rituels collectifs complexes de danse napi. Ces rituels sont effectués à chaque fois que quelqu'un se fait mordre afin d'éviter que cela ne se reproduise.

29. Sapajou jaune en français, une espèce très répandue dans la forêt amazonienne.

30. Overall appelle le suffixe $-k i$ un «transferred action aktionsart» $(2007$, p. $78-81)$, et Peña le traduit par « while moving » $(2015$, p. 629). Ce suffixe appartient au domaine sémantique du « mouvement associé » (Koch 1984 et Guillaume 2006 cités par Peña 2015, 
Anen 2 - Churuwi pinchu et tseem (chanteur: Pedro Chuim)
1) Churuwi pinchuwa, churuwi
Aigle orné, aigle orné pinchuwake
2) Churuwi pinchukekuwa, churuwi
Aigle bien orné, aigle bien orné (pichukeku) $(\times 2)$
3) Yaki, yakintiakekuwa, yaki yakintiakeku
4) Yankukijai churuwi pinchuwa asankekuwa
Haut, très haut dans le ciel, je jaunis, je suis l'aigle orné

5) Yaki, yakintiakeku, yankuki, (yankukijai) Haut, très haut, je jaunis

6) Tuinkiya, tuinki wiyanu imiajunake

D'où? D'où? Je suis le puissant chasseur

7) Yankukitiu, yankukitiu, uwerkatichuna

8) Yankurtukiamatu yankurtatme

9) (Ayantantunmakeshi) $(\times 2)$ tuinki imiatuke

Jaunissant, jaunissant, qui ne laisse pas s'échapper [ses proies]

Tu vas jaunir

10) Yankukijai, yankukijai, yankukijaiyu

En haut? En bas? Où est le puissant chasseur?

Je jaunis, je jaunis, je jaunis

« je jaunis » ou « je deviens jaune », peut être compris de différentes manières, tout à fait complémentaires. Dans le corpus mythique wampis, commun dans les grands traits à celui des autres populations jivaro, Etsa, le Soleil, associé à la couleur jaune, est réputé être un excellent chasseur. C'est Etsa qui est à l'origine des techniques de fabrication de la sarbacane ${ }^{31}$. Il en est de même pour Yankuam, Vénus, nom formé avec la racine yanku / jaune, et pour les étoiles yaa, enfants du félin shiashia ${ }^{32}$ qui ont été capables de tuer ce père grand chasseur. L'aigle, en montant dans le ciel et baigné par la lumière du soleil, apparait de plus en plus jaune à un observateur terrestre. En jaunissant, il devient comme le soleil, très bon chasseur. C'est d'ailleurs quand l'aigle orné vole haut dans le ciel qu'il peut repérer sa proie pour ensuite fondre sur elle. Que le rapace puisse localiser sa proie de si haut est le signe d'une vue excellente et d'un pouvoir prédateur exceptionnellement important. De plus, avoir de bons yeux, un regard pénétrant et imposant est signe pour les Wampis de puissance, de force individuelle. Enfin, quel meilleur exemple de force prédatrice émanant des yeux que ceux de l'aigle orné, perçants et jaunes? « Devenir jaune » fait donc référence à un réseau de savoirs écologiques, physiologiques et mythologiques

p. 629). Il marque un mode d'action caractérisé par un déplacement du sujet de l'action dans l'espace.

31. Voir notamment García-Rendueles 1996 pour les Wampis ou Pellizzaro 1990 pour les Shuar d'Équateur.

32. Espèce non-identifiée. 
qui permettent de générer une disposition affective de prédateur et annoncent une relation cynégétique. Cette couleur était par ailleurs déjà présente dans le premier anen en référence à l'aube, le moment d'aller à la chasse. Au vers 6 , se met en place le « contact» avec la proie par un procédé des plus intéressants. Dans le contexte, les questions tuinkiya, tuinki « D'où? D'où? », peuvent être posées par deux énonciateurs : le chasseur et le petit singe. Le premier cherche sa proie, le deuxième son prédateur. Le chanteur emprunte la voix du singe en même temps qu'il use de la sienne propre. Mon hypothèse est que ce « contact » des voix dans l'énoncé permet celui du chasseur wampis et de sa proie dans l'action cynégétique. C'est une manière pour lui de pénétrer le cœur et les pensées du primate afin qu'aux vers 8 et 9, il s'empare totalement de sa voix et de son comportement: ayant vu l'aigle « jaunir », c'est-à-dire haut dans le ciel et s'apprêtant à plonger, le singe paniqué essaie de situer le danger dans les reliefs des collines de Kampankis. À mesure que l'anentrin devient un puissant chasseur de plus en plus dangereux, la peur de tseem grandit et réciproquement.

Contrairement au chant précédant dans lequel la proie, en quelque sorte « virtuelle », n'est pas encore présente dans l'action cynégétique, dans celui-ci une relation de prédation intense est instituée avec le sapajou. Il ne s'agit plus seulement d'adopter le comportement et l'attitude corporelle du bon chasseur mais de parvenir aussi à ressentir la panique croissante du petit singe se demandant par où l'aigle va attaquer. L'anentrin doit faire l'expérience simultanée des deux dispositions affectives afin de donner corps à la relation de prédation aigle-singe décrite dans le chant.

Pour augmenter ses chances de réussite, le chasseur peut puiser dans son répertoire un chant évoquant une relation de dépendance plutôt qu'une scène de prédation. Pour illustrer ce cas, penchons-nous sur un anen adressé cette fois-ci à un artefact: la sarbacane uum du chanteur.

L'homme vient de s'éveiller, un peu avant le lever du jour, et se prépare à partir à la chasse pendant que sa femme ravive le feu pour y chauffer un peu de bière de manioc. Il a décidé d'utiliser sa sarbacane mais doit la débarrasser de la salive et des saletés accumulées lors de la dernière sortie. Le nettoyage devient alors le moyen de générer une relation de type aîné-cadet avec l'arme. Lorsqu'il s'en saisit, il lui dit de se réveiller aux vers 3 et 11 afin d'aller à la chasse avec le verbe shintiasakia / shintia-sa-kia / se réveiller-ATT-IMP.FAM dans lequel on trouve le suffixe -kia dénotant l'impératif familier toujours à la deuxième personne du singulier, précédé du morphème atténuatif -sa traduit par « doucement ». Il entreprend alors de décrasser l'instrument avec une tige au bout de laquelle est placée une boule de coton uruch, le même qui servira à propulser les fléchettes tsentsak. Il passe ce gros coton-tige par l'extrémité d'où sortira le projectile, un orifice que le chanteur associe à l'œil d'un de ses cadets aux vers $14,15,16$ et 17 . La relation aîné-cadet est déclenchée dès le deuxième vers : 1'arme est désignée par le nom humain Tashararunanu, pour 
Anen 3 - Le chasseur et sa sarbacane (chanteur: Pedro Chuim)

1) Kashikikuwa, kashikiku, kashikikuwa, Tôt le matin kashikikuwa
2) Tashararunanu, tashararuna
[Nom donné à la sarbacane]
3) Aya shintiasakia $(\times 3)$
Réveille-toi doucement
4) Kashikiyau, kashikikuwa
Tôt le matin

5) Aya yatsuchita, yatsuchitu, yatsuchiya

Rien que, petit frère, petit frère, petit frère,

6) Au naintiarana kashikiku

Là-bas dans cette colline, tôt le matin

7) Au naintiarana, naintiarana

Là-bas dans cette colline

8) Ai naintiumumi, naintiumumi,

Allons dans cette colline

naintiumumi

9) kashikiku, kashikikuwa

Tôt le matin

10) Tashararunanu, tashararuna

[Nom donné de la sarbacane]

11) Aya shintiasakia $(\times 2)$

Réveille-toi doucement

12) karinchinme ishi ishimu wajantakia, ishi ishimu wajantakia

$\mathrm{Tu}$ as sommeil, assieds-toi pour t'enlever le sommeil

13) Au naintiarana, naintiarana, ai naintiumumi, naintiumumi, naintiumumi

Là-bas dans cette colline, dans cette colline

14) Aya jiichirmeya epesakia $(\times 2)$ epesakia

15) Muchi japirkutake, tanurkutake jinkimiyu

16) Mitsumsakia $(\times 2)$

Ferme doucement ton petit œil

Lavant ta petite chassie,

Frottant pour y aller

Ferme doucement ton œil

17) Aya muchichirme japirkutake tanurkutake

Lavant seulement ta petite chassie,

Frottant

18) Aya jinkimiyu, jinkimiyara.

Rien que pour y aller, pour y aller

être ensuite appelée au vers 5 « petit frère » avec le diminutif affectueux -chi. Aux vers 3, 11, 12, 14 et 16. L'impératif familier -kia infléchissant toutes les formes verbales dans ces vers manifeste clairement le contrôle que le chanteur exerce sur son cadet-sarbacane. La rudesse du réveil sur le mode impératif est adoucie par l'usage de l'atténuatif -sa dans shintiasakia mais aussi dans epesakia / epe-sa-kia / fermer-ATT-IMP.FAM et son synonyme mitsumsakia / mitsum-sa-kia / fermer-ATT-IMP.FAM. Dans ces deux derniers cas, l'atténuatif marque la bienveillance de l'ainé envers son cadet lui suggérant de « fermer doucement », autrement dit, de faire attention lorsqu'il approche la tige cotonnée de son œil. Par ailleurs, la prolifération du terme aya traduit par « seulement » 
ou « rien que » au vers $3,5,11,14,17$ et $18^{33}$ montre que le chanteur tente de convaincre l'artefact de sortir de son sommeil pour aller à la chasse. Le mot aya permet à l'anentrin de simuler l'exceptionnalité de cet acte cynégétique. Il laisse entendre à son cadet-sarbacane que la tâche est exceptionnelle et qu'il pourra bientôt retourner se reposer.

Cet anen permet de mettre en lumière un phénomène tout à fait intéressant. À ma connaissance, rien dans les mythes ni dans les usages quotidiens ne suggère que les Wampis prêtent des qualités physiques ou psychologiques humaines à la sarbacane. Cela signifie que l'attribution d'une vie humaine à l'artefact est momentanée et qu'elle s'opère par la production volontaire d'une relation affective ressentie par l'anentrin plaçant l'objet dans une position de petit frère; en dehors du temps de l'énonciation de cet anen et de la partie de chasse qui s'ensuit, la sarbacane est un artefact inanimé et insensible. Ce faisant, l'anentrin délègue en partie sa propre capacité à viser correctement l'animal à la sarbacane elle-même qui, avec son « œil », ajustera le tir. Un autre anen s'énonçant au moment du tir, assimile la sarbacane à une espèce de guêpe, la fléchette tsentsak faisant alors office de dard. Les qualités attribuées à l'artefact sont contextuelles et dépendent des objectifs ponctuels que souhaite atteindre l'anentrin au cours de l'action cynégétique : rien n'empêche le chasseur d'énoncer d'abord l'anen « cadet-sarbacane » lors du nettoyage et ensuite de subvocaliser l'incantation " guêpe-sarbacane » lorsque la bête est tenue en joue.

Ces trois anen permettent à l'anentrin de générer une attitude corporelle et des dispositions affectives de bon chasseur. Ils évoquent soit une relation de prédation à établir avec l'animal, soit une relation de dépendance de type aînécadet facilitant le maniement de la sarbacane. Nous allons maintenant analyser deux anen dans lesquels la mobilisation d'une relation de dépendance va servir in fine à accomplir l'acte prédateur. L'anentrin utilise alors un leurre affectif pour attirer le gibier et le mettre en position de proie.

\section{Le leurre affectif}

Le chant magique suivant décrit une relation amoureuse homme-femme entre le chasseur et sa proie afin que l'anentrin puisse leurrer le gibier et l'inciter à s'approcher. Les chasseurs wampis savent distinguer à travers l'épaisse végétation amazonienne les chemins qu'empruntent régulièrement différentes espèces animales. C'est le cas pour le tinamou waa (Crypturellus sp.), un

33. La traduction du terme aya en français est difficile. J'ai choisi de ne pas la faire apparaître aux vers 3 et 11 afin d'éviter la juxtaposition des deux adverbes «seulement» et « doucement». La syntaxe aurait été incorrecte et cela aurait obscurci le sens. Je profite de cette note pour remercier Grégory Deshoullière pour sa relecture des traductions et les précisions qu'il m'a apportées. 
oiseau terrestre, qui après son passage laisse une sorte de couloir de feuilles aplaties, bien évidemment invisible aux yeux du néophyte. Les tinamous, comme beaucoup d'autres animaux, utilisent toujours le même itinéraire tant qu'aucun danger ne s'y présente. À l'endroit où le chemin semble s'estomper, le chasseur chevronné va installer le piège munuk. Il consiste à disposer des feuilles de palmier plantées en cercle en laissant une petite entrée au niveau du chemin. Au niveau de cette entrée, est érigé un piège formé d'un lasso qui se resserrera sur le cou du volatile. Le chasseur se place alors à l'intérieur du cercle en prenant soin de se dissimuler derrière les feuilles, puis chante cet anen.

Anen 4 - Le tinamou waa et le piège munuk (chanteur: Jorge Tsukanka)

1) Ame aishirmeku

2) Wake mesemrachirku

3) Jiiiiitsuatsrua tsrua tsrua untsurmawa

4) Wake mesemrachirku

5) Jikia jikiataku

6) Jiiiiitsuatsrua tsrua tsrua untsurmawa

7) untsurmawa $(\times 2)$

8) Ame aishirmeku

9) Jiiiiitsuatsrua tsrua tsrua untsurmawa

10) untsurmawa $(\times 2)$
Ton mari à toi

[Est] triste

« Jiiiiitsuatsrua tsrua tsrua », il t'appelle

Tout triste

Empli d'un sentiment amoureux

« Jiiiiitsuatsrua tsrua tsrua », il t'appelle

Il t'appelle

Ton mari à toi

" Jiiiiitsuatsrua tsrua tsrua », il t'appelle

il t'appelle

L'anentrin commence ici par se représenter le partenaire amoureux du tinamou aux vers 1 et 2 mais ne s'identifie pas avec lui puisqu'aux vers 3, 6, 7, 9 et 10 le verbe untsurmawa / untsurma-wa / appeler-3sG est conjugué à la troisième personne. Aux vers 2 et 4 est nommée explicitement la tristesse ressentie par le mari en l'absence de son amante gallinacée, que le chanteur doit éprouver grâce au dispositif rituel. La génération de cette disposition affective est renforcée au vers 5 par la mention de la mélancolie et par la profusion du diminutif -chi tout au long du chant évoquant la tendresse. Afin que le leurre affectif soit complet, l'anentrin imite le cri de l'oiseau " jiiiiitsuatsrua tsrua tsrua " pour l'appeler aux vers 3, 6 et 9. L'objectif du chant est d'attirer le tinamou dans le piège posé à l'entrée en suscitant chez elle de la compassion pour son partenaire amoureux esseulé. On comprend mieux pourquoi le chasseur ne s'identifie pas avec le mari dans le chant bien que ce soit lui qui appelle la proie. Nous avons ici une forme de débrayage par laquelle le sujet de l'énonciation se dissocie du sujet de l'action dans l'énoncé. Mon hypothèse est que par cette opération, l'anentrin cache sa position de chasseur derrière la figure de l'époux transi. 
Dans une variante du même chant adressé cette fois-ci au tinamou tsuan (Crypturellus soui), la scène vient s'enrichir d'un nouvel acteur, l'insecte volant

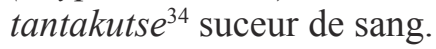

Anen 5 - Le tinamou tsuan et le piège munuk (chanteur: Calixto Tsukanka)

1) Aishirme untsurmawai $(\times 2)$

2) Jiii jirua jirua jirua

3) Untsurmawai juikiakia juikiakia juikiakia

4) Tantakutse $(\times 2)$

5) Shitiatrusia $(\times 2)$

6) Tantakutse $(\times 2)$

7) Jiii jirua jirua

8) Aishirme untsurmawai
Ton mari t'appelle

« Jiii jirua jirua jirua »

Il t’appelle, dépêche-toi

[Nom d'un insecte volant non-identifié]

Pousse [-la] vers moi doucement

[Nom d'un insecte volant non-identifié]

« Jiii jirua jirua »

Ton mari t'appelle

Cet anen mobilise à nouveau une relation de dépendance amoureuse hommefemme pour attirer tsuan dans le piège munuk. L'insecte a pour rôle de malmener la proie et de la pousser à rejoindre son mari qui l'attend impatiemment. Tantakutse reçoit l'ordre au vers 5 d'incommoder la proie mais avec une certaine retenue, que je traduis par l'adverbe « doucement », et encodée par l'atténuatif -sa qui vient fusionner avec l'impératif familier -kia dans shitiatrusia / shitia-tu-ru-sa+kia / pousser-APPL-1SG.OBJ-ATT+IMP.FAM. Le morphème -ru indique que l'énonciateur est concerné par l'action « pousse », ce que je traduis dans le texte par «vers moi ». L'usage de cet atténuatif est particulièrement intéressant car il révèle également la complexité de la relation affective que le chasseur est en train d'établir avec l'oiseau. Le chanteur doit s'efforcer de ressentir la disposition affective de l'époux délaissé afin d'attirer sa conjointe et future proie. Or, en ordonnant à l'insecte de tourmenter, voire de sucer le sang de celle qui doit momentanément être sa femme pour la précipiter dans le piège munuk, le chanteur prend le risque de laisser transparaître ses véritables intentions : étrangler l'oiseau pour en faire sa pitance. C'est pour rendre moins nocive l'action de l'insecte au vers 5 qu'est fait usage de l'atténuatif. Ainsi, le chanteur dissimule son désir prédateur, afin qu'au vers 7 , la proie ne puisse pas déceler la présence du chasseur derrière le cri " jiii jirua jirua ». Le chanteur s'efforce de ressentir la relation de dépendance décrite dans l'anen et masque ainsi la relation cynégétique qu'il souhaite établir.

34. Je n'ai pas pu identifier cet insecte que mon interlocuteur wampis a appelé en espagnol «mosca » (mouche). Je le soupçonne d'être une sorte de taon. 
S'il est vrai que l'efficacité du leurre réside en partie dans la capacité du chasseur à copier les caractéristiques de l'Autre animal - le cri de l'oiseau -, autrement dit dans son « habilité à en retenir et imiter les différences » (Artaud 2013, p. 9), dans ces deux derniers chants, cette efficacité se fonde également sur une propriété que les Wampis estiment avoir en commun avec certains êtres non-humains : l'affectivité. Pour le chasseur wampis, le tinamou possède la capacité d'éprouver un sentiment amoureux. En ce sens, on peut voir dans la construction circulaire du piège munuk, la reproduction d'une maison wampis depuis laquelle un mari appellerait sa femme pour qu'elle rentre vite. Une forme simplifiée du piège existe dans laquelle le chasseur installe le mécanisme à lasso directement sur le chemin, sans cette construction. Le piège munuk n'aurait aucun intérêt sans l'énonciation de cet anen par le chasseur simulant le conjoint à l'intérieur de la maisonnée. La construction circulaire en feuilles de palmier est donc un élément essentiel des conditions pragmatiques de l'énonciation de ces anen. La mise en scène autant extérieure - la fausse maison - qu'intérieure - les représentations et les affects évoqués par le chant - ajoute au leurre sonore " jiii jirua jirua » (pouvant être énoncé à voix haute) une épaisseur affective rendant, aux yeux du chasseur, le dispositif de la tromperie plus efficace.

\section{Conclusion}

Chez les Wampis, l'activité cynégétique inclut un travail affectif sur soi commençant la veille, lorsque l'homme se focalise sur son désir de faire un rêve de bon augure kuntukmamu qui augmentera ses chances de trouver le gibier le lendemain. Le matin, le « ventre vide » aide le chasseur à créer une relation de prédation et/ou de dépendance avec l'animal grâce à la sensation de faim, la souffrance et l'incitation à compatir (en particulier dans le cas du leurre affectif). La concentration sur la relation à établir avec la bête arrive à son paroxysme lors de la récitation des anen et de l'effort shir anentaimat. Penser et ressentir intensément la scène décrite dans les incantations permet aux Wampis d'incarner la sémantique textuelle afin de contrôler le cours de l'action cynégétique. Comprendre la qualité performative attribuée aux anen nous invite à prendre en considération la dimension affective de ces actes de langage silencieux dont les effets pathémiques sur l'allocutaire dépendent de ceux ressentis par le locuteur.

Dans le texte de ces charmes magiques, les affects ne sont pas figés par un substantif (par exemple nous ne trouvons pas de mot traduisant la "peur» du sapajou dans le chant numéro deux), mais sont évoqués par une mise en scène de modèles de relations forgés par la praxis quotidienne (mari-femme; aîné-cadet; aigle orné-sapajou jaune; etc.), auxquels sont associées des actions caractéristiques (voler haut dans le ciel avant de plonger sur la proie, aider son petit frère à se laver, appeler sa femme, etc.). Des marqueurs grammaticaux, 
des figures de rhétorique et autres dispositifs linguistiques permettent d'encoder certains aspects sensibles de la relation que le chanteur désire établir avec les destinataires (possessifs, diminutifs, impératifs, atténuatifs, hypotyposes, etc.) ${ }^{35}$. En invoquant ces interactions, ces chants constituent une forme originale de verbalisation des affects. En somme, les anen offrent l'occasion d'ethnographier une tentative de manipulation des relations entre humains et non-humains, au sein d'un collectif où le contrôle des dispositions affectives des autres passe par une maîtrise de ses propres passions. *

* Manuscrit reçu en mai 2015, accepté pour publication en mai 2017.

\begin{tabular}{|c|c|c|}
\hline+ & $\begin{array}{l}\text { Sépare des morphèmes phonologiquement } \\
\text { identifiables ayant « fusionnés » } \\
\text { (« in "fused" forms », Overall 2007, } \\
\text { p. XVIII; Peña 2015, p. 882). }\end{array}$ & \\
\hline $1,2,3 \ldots$ & $1^{\mathrm{re}}$ personne, $2^{\mathrm{e}}$ personne... & \\
\hline OBJ & Objet & \\
\hline SG & Singulier & \\
\hline APPL & Applicatif & $-t u$ \\
\hline ATTRIB & Attributif & -tinu \\
\hline DECL & Déclaratif & $-i$ \\
\hline EXPL & Explétif & \\
\hline IMP.FAM & $\begin{array}{l}\text { Impératif familier, toujours à } \\
\text { la } 2^{\mathrm{e}} \text { personne du singulier }\end{array}$ & $-k i a$ \\
\hline NZR & Nominalisateur & $-i n u ;-u ;-$ mau ; -tai \\
\hline POSS & Possessif & - rin \\
\hline VZR & Verbalisateur & $-m a$ \\
\hline
\end{tabular}

Tableau des signes, abréviations et morphèmes correspondants.

35. Si dans cet article je me suis concentré sur l'analyse des textes des chants anen, il ne fait nul doute que leur musicalité occupe une place importante au sein du dispositif rituel d'énonciation (voir par exemple les travaux ethnomusicologiques de Salivas 2002 et Bammer de Rodríguez 2015). 


\section{Références citées}

Amodio Emanuele

1988 «Prefacio », in Emanuela Napolitano, Shuar y anent: el canto sagrado en la historia de un pueblo, Abya-Yala, Quito, p. 1-8.

ARTAUd Hélène

2013 «Introduction », in Hélène Artaud (éd), Leurrer la nature, L’Herne, Paris, p. 9-15.

BAMMER DE Rodríguez Nora

2015 «La voz mágica. El ánent shuar como puente sonoro entre los mundos », in Bernd Brabec de Mori, Matthias Lewy et Miguel A. García (dir.), Sudamérica y sus mundos audibles. Cosmologías y prácticas sonoras de los pueblos indigenas, Ibero-Amerikanisches Institut, Berlin, p. 69-82.

BESNIER Niko

1990 «Language and affect», Annual Review of Anthropology, 19 (1), p. 419-451. Boster James S.

2003 «Arutam and cultural change », Antropológica, 99-100, p. 165-185.

BRown Michael F.

1984 «The role of words in Aguaruna hunting magic », American Ethnologist, 11 (3), p. 545-558.

1985 Tsewa's gift: magic and meaning in an Amazonian society, Smithsonian Institution Press, Washington.

Chaumeil Jean-Pierre

1993 «Des Esprits aux ancêtres. Procédés linguistiques, conception du langage et de la société chez les Yagua de l'Amazonie péruvienne ", L'Homme, 126-128, p. 409-427.

2010 « Des sons et des esprits-maîtres en Amazonie amérindienne », Ateliers d'anthropologie [en ligne], 34, https://doi.org/10.4000/ateliers.8546, consulté le $05 / 12 / 2017$.

Costa Luiz

2010 «The Kanamari body-owner. Predation and feeding in Western Amazonia », Journal de la Société des américanistes, 96 (1), p. 169-192.

Descola Philippe

1986 La nature domestique: symbolisme et praxis dans l'écologie des Achuar, Maison des sciences de l'homme, Paris.

1989 «Head-Shrinkers Versus Shrinks: Jivaroan Dream Analysis », Man, New Series, 24 (3), p. 439-450.

1993a Les lances du crépuscule: relations jivaros, Haute-Amazonie, Plon, Paris.

1993b « Les Affinités sélectives Alliance, guerre et prédation dans l'ensemble jivaro », L'Homme, 126-128, p. 171-190.

Fausto Carlos

2008 « Donos demais: maestria e domínio na Amazônia », Mana, 14 (2), p. 329-366.

García-Rendueles Manuel

1996 "Yaunchuck»: universo mítico de los Huambisas, Kanus (Río Santiago), Centro Amazónico de Antropología y Aplicación Práctica, Lima. 
GNERre Maurizio

2009 «While I sing I am sitting in a real aiplane. Innovative contents in Shuar and Achuar ritual communication », in Gunter Senft et Ellen B. Basso (éd.), Ritual Communication, Berg, Oxford, p. 293-316.

Guillaume Antoine

2006 « La catégorie du "mouvement associé" en cavineña: apport à une typologie de l'encodage du mouvement et de la trajectoire », Bulletin de la Société de linguistique de Paris, 101 (2), p. 415-436.

Gutierrez Choquevilca Andrea-Luz

2012 Voix de "maîtres » et chants d'oiseaux: pour une étude pragmatique de l'univers sonore et la communication rituelle parmi les Quechua d'Amazonie péruvienne, thèse de doctorat en ethnologie, université Paris-Ouest Nanterre La Défense.

2013 «Face-à-face interspécifique et pièges à penser Quechua de Haute Amazonie (Pastaza) », in Hélène Artaud (éd.), Leurrer la nature, L'Herne, Paris, p. 33-47.

HARNER Michael

1972 The Jivaro, people of the sacred waterfalls, American Museum of Natural

Koch Harold History Doubleday/Natural History Press, New York.

1984 "The category of "associated motion" ", Kaytej. Language in Central Australia, 1, p. 23-34.

Lutz Catherine et Lila ABu-Lughod (dir.)

1990 Language and the politics of emotion, Cambridge University Press, Cambridge.

Lutz Catherine et Geoffrey M. White

1986 «The Anthropology of Emotions », Annual Review of Anthropology, 15, p. 405-436.

MADER Elke

1999 Metamorfosis del poder: persona, mito y visión en la sociedad shuar y achuar (Ecuador, Perú), Abya-Yala, Quito.

Massumi Brian

2002 Parables for the virtual: movement, affect, sensation, Duke University Press, Durham.

NAPOLITANO Emanuela

1988 Shuar y anent: el canto sagrado en la historia de un pueblo, Abya-Yala, Quito. Overall Simon E.

2007 A Grammar of Aguaruna, thèse de doctorat en philosophie, La Trobe University, Melbourne.

Pellizzaro Siro M.

1990 Arutam: mitología shuar, Abya-Yala, Quito.

PeÑa Jaime G.

2015 A Grammar of Wampis, thèse de doctorat en philosophie, University of Oregon, Eugene.

Reichel-Dolmatoff Gerardo

1973 Desana: le symbolisme universel des Indiens Tukano du Vaupés, Gallimard, Paris. 
RosAldo Michelle Z.

1984 « Toward an anthropology of self and feeling », in Richard A. Shweder et Robert A. LeVine (éd.), Culture theory. Essays on mind, self, and emotion, Cambridge University Press, Cambridge, p. 137-158.

Rubenstein Steven L.

2012 « On the importance of visions among the Amazonian Shuar », Current Anthropology, 53 (1), p. 39-79.

SALIVAS Pierre

2002 Musiques jivaro. Une esthétique de l'hétérogène, thèse de doctorat en musicologie, université Paris 8.

Seymour-Smith Charlotte

1988 Shiwiar: identidad étnica y cambio en el Río Corrientes, Abya-Yala, Quito.

STEEL Daniel

1999 «Trade goods and Jívaro warfare. The Shuar 1850-1957, and the Achuar, 1940-1978 », Ethnohistory, 46 (4), p. 745-776.

SURRALLÉs Alexandre

2000 «La passion génératrice », L’Homme, 154-155, p. 123-144.

2003 Au coeur du sens. Perception, affectivité, action chez les Candoshi, CNRS Éditions/Éditions de la Maison des Sciences de l'Homme, Paris.

TAYLOR Anne-Christine

1985 «L'art de la réduction. La guerre et les mécanismes de la différenciation tribale dans la culture jivaro », Journal de la Société des américanistes, 71 (1), p. 159-173.

1997 «L'oubli des morts et la mémoire des meurtres. Expériences de l'histoire chez les Jivaro », Terrain, 29, p. 83-96.

2000 « Le sexe de la proie. Représentations jivaro du lien de parenté », L'Homme, 154-155, p. 309-334.

2003 «Les masques de la mémoire. Essai sur la fonction des peintures corporelles jivaro », L'Homme, 165, p. 223-248.

TAYLOR Anne-Christine et Ernesto CHAU

1983 « Jivaroan magical songs. Achuar anent of connubial love », Amerindia, 8, p. $87-127$.

THRIFT Nigel

2008 Non-representational theory. Space, politics, affect, Routledge, London.

Yvinec Cédric

2005 «Que disent les tapirs? De la communication avec les non-humains en Amazonie », Journal de la Société des américanistes, 91 (1), p. 41-70. 
